\title{
Keynote: \\ For a Few Triples More
}

\author{
Gerhard Weikum \\ Max-Planck-Institute for Informatics (MPI), DE
}

\begin{abstract}
The Web of Linked Data contains about 25 billion RDF triples and almost half a billion links across data sources; it is becoming a great asset for semantic applications. Linked Data comprises large general-purpose knowledge bases like DBpedia, Yago, and Freebase, as well as many reference collections in a wide variety of areas, spanning sciences, culture, entertainment, and more. Notwithstanding the great potential of Linked Data, this talk argues that there are significant limitations that need to be overcome for further progress. These limitations regard data scope and, especially, data quality. The talk discuss these issues and approaches to extending and enriching Linked Data, in order to improve its scope, quality, interpretability, cross-linking, and usefulness.
\end{abstract}

